\title{
EDUCAÇÃO BRASILEIRA: ANÁLISE TEMÁTICA (1991-1994)
}

\author{
Maria Pia Giazzi NASSRI \\ Universidade Mogi das Cruzes \\ Marisa Bueno Mendes GARGANTINI \\ PUC-Campinas \\ Regina Coeli Bezerra de Melo NASSRI \\ Universidade Mogi das Cruzes
}

\section{RESUMO}

\begin{abstract}
Analisa a freqüência de aparecimento dos temas e dos nomes das universidades brasileiras, oficiais e particulares, nos artigos da Revista Educação Brasileira, publicada pelo Conselho de Reitores, no período de 1991 a 1994. Constata que a análise permitiu uma visão geral dos problemas educacionais do país no período, que houve diferença significativa entre as categorias de temas, prevalecendo a Genérica e que a produção dos docentes das universidades estatais destacou-se significantemente. Conclui pela relevância da publicação enquanto identifica $e$ analisa a problemática da educação superior brasileira.
\end{abstract}

Palavras-chave: periódico científico, temas, educação superior brasileira.

\section{ABSTRACT}

This study analyzes the frequency of themes and names of the Brazilian official and private universities in the papers of the journal Brazilian Education, published by the Council of Rectors, has from 1991 to 1994. It verifies that the analysis allowed a general vision of the educational problems of the country in that period, that there was a significant difference among the categories of themes, prevailing the Generic and that the papers written by the teachers of the public universities have increased significantly. The results have also pointed out the relevance of the publication because it identifies and analyzes the problem of the Brazilian higher education.

Keyword: scientific journal, themes, Brazilian higher education.

\section{INTRODUÇĀO}

A sociedade, em vertiginosa transformação, espera que a universidade possa rever suas práticas educativas e reformular seus objetivos tendo em vista a competente realização de seu papel social.

A universidade tem sido concebida como "espaço institucional de pesquisa” (STUMPF, 1994, 
p. 45), formando com o ensino e a extensão uma unidade que deveria ser entendida como indissolúvel.

Segundo DURHAN (1994), a educação superior foi idealizada como uma organização que deveria buscar o "modelo" de perfeição em seu desenvolvimento. Assim, a universidade pretendida deveria estender-se a todos os campos de conhecimento, unindo ensino e pesquisa, sendo, pois, a instituição que ao mesmo tempo que cria o conhecimento deve torná-lo acessível a todos.

No final dos anos sessenta, quando o Brasil buscava a inovação e transformação do ensino superior e das universidades, foi introduzida, como fator indispensável de desenvolvimento, a pesquisa. É conveniente assinalar que os resultados de pesquisa, apesar de serem considerados altamente relevantes como realização pessoal, representam um papel considerável no domínio social. Um aspecto que, portanto, não pode ser desconsiderado, é a possibilidade das informações serem acessadas e utilizadas pelos membros da comunidade acadêmica e por outros que se interessem pelo assunto. Para que a sociedade possa participar dos avanços científicos, tecnológicos e dos benefícios que as pesquisas trazem, é necessário que a divulgação dos resultados se faça por meio de publicações especializadas, possibilitando, assim, a propagação da informação (WITTER, 1990b).

A pesquisa documental realiza o levantamento de dados relativos às publicações já concluídas, permitindo o conhecimento de informações disponíveis em vários assuntos. $\mathrm{O}$ conhecimento elaborado, a partir do levantamento da produção existente, facilita, conforme WITTER (1990a), o trabalho do pesquisador iniciante, enquanto detecta temas, hipóteses e problemas, possibilitando, assim, o conhecimento pretendido.

O pesquisador, ao buscar informação para o embasamento de seu trabalho, pode encontrar indícios significativos em levantamentos envolvendo títulos de pesquisas já realizadas. É necessário ressaltar a importância de uma perfeita elaboração dos títulos dos trabalhos científicos, pois, o título "constitui uma estrutura que dá acesso ao conteúdo dos trabalhos" (SODEK, 1997, p.82), sendo o primeiro contato do leitor com o texto.

As discussões, as novas idéias e as descobertas apresentadas nas reuniões científicas, em geral, são, também, conhecidas por meio dos periódicos. Os periódicos são fontes de informações, fruto das pesquisas que rapidamente chegam aos usuários das ciências. São publicações ágeis que enfocam temas apresentando novos conceitos científicos; servem como meio oficial de registro científico público e, também, como veículo formal de comunicação da informação científica (CASTRO, 1992).

O Conselho de Reitores, acreditando que estas publicações têm papel muito importante na difusão das pesquisas e no desenvolvimento do conhecimento, criou o seu periódico para análise dos problemas no campo da educação superior brasileira, não perdendo de vista os estudos que ocorrem no contexto universitário mundial.

O Conselho de Reitores das Universidades Brasileiras é uma sociedade sem fins lucrativos que foi criada em 1966. Sua finalidade é integrar as universidades buscando o aperfeiçoamento do ensino superior do país. Assim, ela se propõe desenvolver o intercâmbio entre universidades brasileiras no intuito de propiciar soluções para os problemas que envolvem a educação superior no Brasil. O Conselho reúne como membros efetivos todos os Reitores das Universidades brasileiras e como membros honorários os ex-Reitores com reuniões ordinárias uma vez ao ano. Os órgãos que compõem a estrutura do Conselho de Reitores são: Plenário, Presidência, Conselho Deliberativo, Conselho Fiscal e Secretaria Executiva, cada um deles com suas atribuições específicas (Estatuto do Conselho de Reitores, 1994)

O Conselho de Reitores, reconhecendo a importância de abrir um espaço para a discussão de temas relevantes sobre educação superior, publica a revista Educação Brasileira há mais de quinze anos. Desde 1978 o periódico é publicado trazendo, à luz de análises críticas, as principais tendências da educação brasileira. É necessário ressaltar a importância dos temas por ele tratados no conhecimento da problemática que o ensino superior brasileiro envolve.

O ensino superior no Brasil, seguindo tendências mundiais, expandiu-se consideravelmente, ocasionando com isto um "status quo" que exige uma ampla análise e 
discussão dos problemas emergentes, na tentativa de reconstrução nacional.

Ele é fruto de mudanças, às vezes drásticas, na sua estrutura e concepção (TIRAMONTE, 1992; TARAPANOFF, 1997; MIRANDA, 1997).

Em 1961, com a primeira Lei de Diretrizes e Bases de Educação Nacional, o então Conselho Federal de Educação decidiu sobre o funcionamento e expansão das escolas federais isoladas, escolas particulares e reconhecimento de universidades. No âmbito de sua jurisdição, os Conselhos Estaduais participavam também da criação de cursos e universidades.

Em 1968, a reforma universitária estabeleceu que o ensino superior, indissolúvel da pesquisa e da extensão, deveria ser ministrado em universidades e, excepcionalmente, em institutos isolados. Teoricamente, a pesquisa passa a ser meta do modelo organizacional. Esta reforma antecipou a do ensino de $1^{\circ}$ e $2^{\circ}$ graus ocorrida em 1971, que trouxe para o Brasil mudanças principalmente quantitativas também no ensino superior (Lei 9394/96 - Dornas, 1997; BRSEZUISKI, 1997).

Em 1988, a nova Constituição Brasileira provocou a criação da Lei de Diretrizes e Bases de Educação Nacional; em relação ao ensino superior, ela apresenta novas diretrizes relativas a: avaliação periódica dos cursos e das universidades; mudança nos exames vestibulares; exigência na titulação docente; possibilidade do ensino à distância; universidades especializadas por campo do saber; definindo ainda que a formação dos profissionais que atuam em todos os níveis de ensino seja feita, de preferência, em nível superior.

A problemática, que hoje as universidades vivem, exige uma análise ampla e séria e, naturalmente, deve envolver os dirigentes e todos que militam nesse nível de ensino.

A pesquisa tem, como papel primordial, não somente o conhecimento da realidade brasileira em suas "deficiências e inadequações" (MARTINS e GALVÃO, 1994), mas, principalmente, no tornar possível o funcionamento eficiente do sistema educacional do Brasil. A produção científica tem a finalidade de promover a inovação (HUBERMAN, 1972), a mudança, necessitando, para isto, da divulgação do conhecimento científico e tecnológico. Os periódicos, publicações que realizam esta tarefa, assumem então uma enorme importância na promoção da rápida expansão do conhecimento produzido.

Neste contexto, a presente pesquisa teve por objetivo a análise dos temas e a freqüência do aparecimento do nome das universidades brasileiras, enquanto ligadas aos autores dos artigos apresentados na revista Educação Brasileira do Conselho de Reitores das Universidades Brasileiras, no período de 1991 a 1994.

\section{MÉTODO}

\section{Material}

O material utilizado consistiu dos exemplares da revista Educação Brasileira do Conselho de Reitores das Universidades Brasileiras.

Ela tem o formato retangular medindo 0,22 $\mathrm{m}$ de altura por $0,15 \mathrm{~m}$ de largura; apresenta capa na cor branca com pigmentos bege-acinzentados, na lateral direita se destaca uma faixa onde se vê um amaranhado de letras nas mesmas cores já descritas. Na parte superior da capa, atravessando a faixa lateral, aparece em vermelho o título da revista: Educação Brasileira; logo abaixo, em uma faixa branca aparecem em letras pretas: "Revista do Conselho de Reitores das Universidades Brasileiras" e na parte inferior, em uma faixa vermelha, aparecem escritos em branco, os dados relativos a volume, número, mês e ano.

Educação Brasileira apresenta em todas as unidades, na página número 4 , o número de indexação, número do volume, número da revista, mês e ano. Apresenta Índices-Sumários (IBICT) além do endereço do CRUB. Do Sumário constam: Editorial, Tema, Textos, Debates, Informativos Bibliográficos e Documentação. A revista Educação Brasileira é publicada semestralmente.

\section{Procedimento}

Foram levantados os artigos apresentados pela revista Educação Brasileira, no período de 1991 a 1994. A partir dos títulos dos artigos foi realizada a classificação dos temas, independentemente, por dois juízes. Após esta 
classificação, foi elaborada uma tabela comparativa, onde foram observadas as relações existentes e os índices de concordância, sendo os mesmos satisfatórios. Posteriormente, foi definida a escolha de uma das classificações, a qual passou a ser objeto da análise aqui apresentada.

\section{RESULTADOS E DISCUSSĀO}

Após estudo dos temas e confronto entre as classificações apresentadas pelo Juiz 1 e Juiz 2, verificou-se $75 \%$ de concordância entre elas (Tabela 1)

A seguir, foi feita escolha aleatória da proposta a ser trabalhada e esta recaiu na classificação do Juiz 1.

Foram definidos 13 temas sínteses:

Administração em universidades: entendida como um complexo conjunto de atividades que se caracterizam como meios para se atingir a eficiência do processo educacional. Exemplo: $A$
Administração de Universidades Públicas. A racionalidade, a ineficiência (SCHWARZMAN, 1991).

Avaliação institucional: fator indispensável no controle de qualidade, enquanto as instituições buscam a eficiência do processo. Exemplo: Evaluación y Financiamiento de la Educación Superior en América Latina: Bases para um nuevo contrato (BRUNNER, 1993).

Cidadania: formação da pessoa enquanto ser participante do seu momento histórico. Exemplo: Cidadania: Dos conceitos à realidade brasileira (LANDO, 1992).

Ciência: a investigação e criação de novos campos de conhecimento. Exemplo: Ciência e responsabilidade intelectual (PELUSO, 1993).

Corpo discente: principal elemento no processo educacional. Exemplo: As condições de vida e de trabalho dos alunos do ensino superior brasileiro: o caso das universidades de Fortaleza (PAUL e RIBEIRO, 1991).

Tabela 1. Temática dos Artigos

\begin{tabular}{|l|c|c|c|c|}
\hline \multicolumn{1}{c|}{ Temas } & F & \multicolumn{2}{c|}{ Juízes } & \% \\
\hline Admin. de Universidades & 2 & $\mathrm{X}$ & $\mathrm{X}$ & $100 \%$ \\
\hline Avaliação Institucional & 2 & $\mathrm{X}$ & $\mathrm{X}$ & $100 \%$ \\
\hline Cidadania & 2 & $\mathrm{X}$ & $\mathrm{X}$ & $100 \%$ \\
\hline Ciência 1 & $\mathrm{X}$ & & & $50 \%$ \\
\hline Corpo Discente & 2 & $\mathrm{X}$ & $\mathrm{X}$ & $100 \%$ \\
\hline Educação Superior & 2 & $\mathrm{X}$ & $\mathrm{X}$ & $100 \%$ \\
\hline Ética 1 & & $\mathrm{X}$ & & $50 \%$ \\
\hline Financiamentos & 1 & & $\mathrm{X}$ & $50 \%$ \\
\hline Formação de Recursos Humanos & 2 & $\mathrm{X}$ & $\mathrm{X}$ & $100 \%$ \\
\hline Graduação & 2 & $\mathrm{X}$ & $\mathrm{X}$ & $100 \%$ \\
\hline Investimentos & 2 & $\mathrm{X}$ & $\mathrm{X}$ & $100 \%$ \\
\hline Outros 1 & & $\mathrm{X}$ & & $50 \%$ \\
\hline Pesquisa & 2 & $\mathrm{X}$ & $\mathrm{X}$ & $100 \%$ \\
\hline Pós-Graduação & 1 & $\mathrm{X}$ & & $50 \%$ \\
\hline Qualidade e Inovação & 2 & $\mathrm{X}$ & $\mathrm{X}$ & $100 \%$ \\
\hline Universidades & 2 & $\mathrm{X}$ & $\mathrm{X}$ & $100 \%$ \\
\hline Total & & & & $\mathbf{7 5 \%}$ \\
\hline
\end{tabular}


Educação superior: entendida como campo de conhecimento e de formação dos profissionais que vão atuar nos mais diferentes campos de trabalho. Exemplo: Crise dos paradigmas da educação superior (DEMO, 1994).

Formação de recursos humanos: a formação de professores pensada em diferentes dimensões; uma que envolve as disciplinas específicas e outra que envolve a formação pedagógica, a qual exige, além do conhecimento metodológico, uma relação de comunicação com o campo de trabalho externo. Exemplo: A formação de docentes: o confronto necessário professor $x$ academia (GATTI, 1992).

Graduação: nível de ensino superior onde se graduam os profissionais nos vários campos do conhecimento. Exemplo: Ensino de Graduação (COELHO, 1994).

Investimento: recursos materiais utilizados no desenvolvimento dos vários setores. Exemplo: Vale a pena investir em educação? (CASTRO, 1991).

Pesquisa: atividade essencialmente humana, orientada no sentido da construção do conhecimento. Exemplo: Condicionamento da qualidade da pesquisa na universidade (DURHAN, 1994).

Pós-graduação: nível de ensino destinado à capacitação de pesquisadores e especialistas de alto nível. Exemplo: Graduação e a capacitação docente (MEDINA,1993).

Qualidade e inovação: a busca da eficiência no processo educacional. Exemplo: Impacto de la investigación en los processos de innovación (AGUERONDO, 1992).

Universidade: instituição onde são desenvolvidas as atividades de ensino, pesquisa e extensão. Exemplo: Produção de ensino na universidade: seara de desencontros (MAROSINI, 1994).

A partir da classificação dos artigos que compuseram a tabela apresentada, estabeleceu-se a elaboração das porcentagens relativas ao número de artigos relacionados, conforme Tabela 2.

Pela análise e classificação dos temas apresentados nos 69 artigos de Educação
Brasileira, observou-se que a maior preocupação dos estudiosos concentra-se nos problemas que envolveram a "Universidade" com $30 \%$ do total dos estudos realizados, enfocando vários aspectos, dentre outros: a atual crise, o problema da autonomia, a produção de ensino, a interdisciplinaridade, a tecnologia etc.

Tabela 2. Incidência dos temas nos artigos

\begin{tabular}{|l|l|l|}
\hline \multicolumn{1}{|c|}{ Temas } & F & \% \\
\hline Admin. de Universidades & 2 & $2,8 \%$ \\
\hline Avaliação Institucional & 4 & $5,3 \%$ \\
\hline Cidadania & 3 & $4,3 \%$ \\
\hline Ciência & 2 & $2,8 \%$ \\
\hline Corpo Discente & 2 & $2,8 \%$ \\
\hline Educação Superior & 8 & $11,5 \%$ \\
\hline Formação Recursos Humanos & 5 & $7,2 \%$ \\
\hline Graduação & 2 & $2,8 \%$ \\
\hline Investimentos & 5 & $7,2 \%$ \\
\hline Pesquisa & 6 & $8,6 \%$ \\
\hline Pós-Graduação & 5 & $7,2 \%$ \\
\hline Qualidade e Inovação & 5 & $7,2 \%$ \\
\hline Universidades & 20 & $30,0 \%$ \\
\hline Total & $\mathbf{6 9}$ & $\mathbf{1 0 0} \%$ \\
\hline
\end{tabular}

O segundo tema, em número de artigos apresentados, foi a "Educação Superior" (11,5\%), na qual o problema aparece tratado sob diferentes pontos de vista: a crise dos paradigmas, a modernidade, a expansão, a L.D.B., entre outros.

O tema Pesquisa representou $8,6 \%$ no total dos artigos apresentados, seguido de Formação de Recursos Humanos, Investimentos, Pós-Graduação, Qualidade e Inovação, cada um, respectivamente, $\operatorname{com} 7,2 \%$ dos trabalhos.

Os artigos sobre Avaliação Institucional representaram 5,3\% do total e Cidadania, 4,3\%.

Os artigos sobre a Administração de Universidades, Ciência, Corpo Discente e Graduação representaram, cada um, 2,8\% do total dos temas apresentados.

É de certa forma incompreensível que em um periódico publicado por órgão que reúne os 
dirigentes das universidades brasileiras, temas como Administração de Universidades, Corpo Discente, Graduação e Ciências, tenham sido tão pouco analisados no período de 1991 a 1994 . O tema Administração de Universidades, provavelmente, hoje, nas atuais publicações, receba tratamento diferenciado, em virtude da busca da gestão de qualidade que ocorre nas instituições, em geral, nesse final de século. Corpo Discente e Graduação não foram alvo de grandes pesquisas o que sugere que as universidades, de um modo geral, interessam-se muito mais pelo nível de pós-graduação e extensão, deixando em segundo plano a graduação e o corpo discente. Atualmente, devido às avaliações que estão sendo postas em prática pelo Ministério de Educação e Desporto, talvez ocorra uma inversão nas preocupações demonstradas pelas instituições universitárias com relação a esse nível de ensino. O tema Ciência talvez possa, nas publicações analisadas, estar integrado a outros, pois, isoladamente, apareceu de forma bastante tímida $(2,8 \%)$.

Considerando a necessidade de viabilizar uma análise mais completa, face à grande dispersão, os temas foram aglutinados em categorias:

Genérica que incluiu Universidade, Educação Superior e Cidadania.

Administração que incorporou Administração, Avaliação, Investimento e Qualidade e Inovação.

Ensino que implicou em Corpo Discente, Graduação e Formação de Recursos Humanos.

Pesquisa que aglutinou os temas Ciência, Pesquisa e Pós-graduação.

A síntese aparece na Tabela 3

Da aglutinação dos temas em categorias foram obtidos os seguintes resultados: a categoria

Tabela 3. Aglutinação dos temas em categorias

\begin{tabular}{|l|r|l|}
\hline \multicolumn{1}{|c|}{ Categorias } & F & \% \\
\hline Genérica & 31 & $45 \%$ \\
\hline Administração & 16 & $23,2 \%$ \\
\hline Ensino & 9 & $13 \%$ \\
\hline Pesquisa & 13 & $18,8 \%$ \\
\hline Total & $\mathbf{6 9}$ & $\mathbf{1 0 0 \%}$ \\
\hline
\end{tabular}

Genérica ficou com 45\%, Administração com 23,2\%, Ensino com 13\% e Pesquisa com 18,8\%, respectivamente.

Tomando-se como nível de significância 0,05 , o número de graus de liberdade $=3 \mathrm{e} \mathrm{x}_{\mathrm{c}}{ }^{2}=7,81$, o que permite rejeitar a hipótese nula no nível 0,05, tendo sido encontrado $x_{0}{ }^{2}=15,35$, concluiu-se que há diferença significativa entre as categorias sendo a Genérica a que prevaleceu.

A segunda parte da pesquisa mostra, no levantamento da freqüência, o aparecimento dos nomes das universidades brasileiras enquanto ligadas aos autores dos artigos apresentados na revista Educação Brasileira, conforme Tabela 4.

Notou-se pelo que demonstra a Tabela 4 , que 13 das 19 universidades representadas, com artigos publicados no periódico Educação Brasileira, são instituições federais, 3 são mantidas pelo poder público estadual (duas das quais pelo Estado de São Paulo e uma pelo Estado de Sta. Catarina), e 3 são instituições particulares (duas do Estado de São Paulo e uma do Rio Grande do Sul).

Portanto, as universidades federais representam $68,6 \%$ das instituições relacionadas, enquanto as estaduais e as particulares representam, cada uma delas, $15,7 \%$, sendo o total $31,4 \%$. As universidades particulares tiveram uma pequena representação nos artigos publicados pela revista Educação Brasileira, provavelmente, por motivos bem diversos. As mais novas, provavelmente, estão tentando se estruturar no campo da pesquisa, não se preocupando ainda com os veículos de divulgação científica enquanto que as universidades particulares tradicionais possuem suas publicações próprias, fato que ocorre também nas instituições oficiais do sistema estadual.

O fato de se ter maior número de publicações das entidades federais, no periódico publicado pelo Conselho de Reitores, talvez, possa ser explicado pela maior disponibilidade de informações que o sistema federal dispõe, possibilitando assim uma via direta de mão dupla com as suas universidades.

Assinalou-se um maior número de artigos publicados pelas universidades federais, havendo uma razão clara para isto, ou seja, em todos os estados brasileiros existem universidades mantidas pelo governo federal e essas escolas mantém uma certa hegemonia com relação ao ensino superior no 
Brasil. As universidades estaduais, que em São Paulo têm grande prestígio, apareceram de maneira tímida, assim como as particulares cuja

Tabela 4. Universidades e Autores representação maior é de universidades tradicionalmente conhecidas como entidades confessionais.

\begin{tabular}{|l|c|c|}
\hline \multicolumn{1}{|c|}{ Universidades } & No Autores & $\%$ \\
\hline Universidade Nacional de Brasília (UNB) & 7 & $15,5 \%$ \\
\hline Universidade de São Paulo (USP) & 5 & $11 \%$ \\
\hline Universidade Federal de Minas Gerais (UFMG) & 5 & $11 \%$ \\
\hline Universidade Federal do Rio Grande do Sul (UFRGS) & 4 & $9 \%$ \\
\hline Universidade Federal do Ceará (UFC) & 4 & $9 \%$ \\
\hline Universidade Estadual de Campinas (UNICAMP) & 3 & $6,5 \%$ \\
\hline Universidade Federal de Juiz de Fora (UFJF) & 3 & $6,5 \%$ \\
\hline Pontifícia Universidade Católica de São Paulo (PUC/SP) & 2 & $4,5 \%$ \\
\hline Pontifícia Universidade Católica de Campinas (PUC-Campinas) & 2 & $4,5 \%$ \\
\hline Universidade Federal de Sta. Catarina (UFSC) & 2 & $4,5 \%$ \\
\hline Universidade Federal de São Carlos (UFSCAR) & 1 & $2 \%$ \\
\hline Universidade Federal de Pelotas (UFPEL) & 1 & $2 \%$ \\
\hline Universidade Federal de Goiás (UFG) & 1 & $2 \%$ \\
\hline Universidade de Caxias do Sul & 1 & $2 \%$ \\
\hline Universidade Federal de Pernambuco (UFP) & 1 & $2 \%$ \\
\hline Universidade Federal do Rio de Janeiro (UFRJ) & 1 & $2 \%$ \\
\hline Universidade Federal de Viçosa & 1 & $2 \%$ \\
\hline Universidade Federal do Paraná (UFPr) & 1 & $2 \%$ \\
\hline Universidade do Estado de Sta. Catarina (UDESC) & $\mathbf{4 6}$ & $\mathbf{1 0 0} \%$ \\
\hline Total & $2 \%$ \\
\hline
\end{tabular}

Contudo, comparando-se em termos de proporcionalidade de categorias de universidades e de artigos delas originados, observou-se que, em média, as universidades federais publicaram 2,4 artigos, as estaduais publicaram 3 artigos e as particulares, 1,6 artigos. Assim, a contribuição das universidades estaduais, proporcionalmente, foi maior do que a das federais e a das particulares.

Com a aglutinação das universidades oficiais: federais e estaduais e as universidades particulares, os resultados relacionados ao número de autores dos artigos apresentados pela revista Educação
Brasileira, ficaram assim definidos; as escolas chamadas públicas com $93,3 \%$ das publicações, e as particulares com $6,7 \%$ conforme aparece na Tabela 5 .

Tabela5. Aglutinação por tipo de Universidade:oficiais e particulares

\begin{tabular}{|l|c|c|}
\hline \multicolumn{1}{|c|}{ Universidades } & No de Autores & \multicolumn{1}{c|}{$\%$} \\
\hline Oficiais & 43 & $93.3 \%$ \\
\hline Particulares & 3 & $6,7 \%$ \\
\hline Total & $\mathbf{4 6}$ & $\mathbf{1 0 0 \%}$ \\
\hline
\end{tabular}


O número de publicações das entidades oficiais é significativamente maior comparado ao das universidades particulares. Neste caso, o número de graus de liberdade $=1$, o nível de significância $0,05, \mathrm{x}_{0}{ }^{2}=3,84$ e foi obtido $\mathrm{x}_{\mathrm{c}}{ }^{2}=19,08$, concluindo-se que não há rejeição da hipótese nula, ou seja, é significantemente mais presente a produção de docentes das universidades estatais (federais + estaduais).

Salienta-se que se pretendeu neste artigo analisar apenas os trabalhos dos representantes das universidades brasileiras, embora a revista Educação Brasileira apresente outros artigos, cujas instituições estão relacionadas, a seguir, apenas à guisa de informação. As instituições representadas são: UNESCO, SBPC, CNPq, Senado Nacional, Secretaria Nacional de Cultura, Presidente de Empresa Particular, IPEA e as Universidades do Chile, Argentina e Alemanha.

\section{CONCLUSÕES}

A pesquisa documental, realizada sobre o periódicoEducação Brasileira, permitiu uma visão geral dos problemas educacionais do Brasil, no período de 1991 a 1994. Os dados mostraram que a tendência tem sido publicar trabalhos genéricos mais do que os que atendem a solução de problemas específicos, provavelmente em decorrência do órgão responsável pelo periódico. Embora seja maior o número de universidades particulares no Brasil, foram as estatais que responderam pela maior parte das publicações. Isto pode se ligar ao fato de só recentemente se estar instalando a pesquisa nas universidades não estatais e também pelo gerenciamento do próprio órgão.

É importante, ao mesmo tempo, ressaltar o papel do Conselho de Reitores nas iniciativas de discutir e apoiar as ações voltadas para o desenvolvimento das universidades brasileiras. A necessidade de mudanças educacionais é cada vez mais urgente e, em vistadisso, cientes de seu papel, as universidades realizam pesquisas científicas que servem de base para a inovação do sistema nacional de educação (DEMO, 1996).

Entretanto, para que os resultados das pesquisas possam ser conhecidos pelos estudiosos, é necessária a rápida divulgação dos estudos realizados. Desta forma, o Conselho de Reitores, ao publicar seu periódico, abre um campo de oportunidades para apresentação de idéias, discussões, reflexões e questionamentos sobre as estruturas institucionais no campo da educação brasileira.

\section{REFERÊNCIAS BIBLIOGRÁFICAS}

BRASIL. Conselho de Reitores das Universidades Brasileiras. Estatuto do Conselho de Reitores das Universidades Brasileiras. Brasília, 1994.

BRASIL. Ministério de Educação e Desporto. Lei de Diretrizes e Bases da Educação Nacional 9394 de 20 de dezembro de 1996. Brasília, 1996.

Estatuto do Conselho de Reitores das Universidades Brasileiras. 1994.

BRSEZUISKI, Iria.LDB interpretada: diversos olhares se entre cruzam. São Paulo: Cortez, 1997.

CASTRO, Maria Helena de. Produção científica dos docentes da escola Superior de Lavras - análise quantitativa. Dissertação de Mestrado. Campinas: PUC-Campinas, 1992.

DEMO, Pedro.Educação e qualidade. $3^{a}$ ed. Campinas: Papirus, 1996.

DORNAS, Roberto. Diretrizes e Bases da Educação Nacional - Comentários e Anotações. Belo Horizonte: Modelo Editorial, 1997.

DURHAM, Eunice. Condicionantes da qualidade de pesquisa na universidade. Educação Brasileira, Brasília, v.16, n.33, p. 31-41, 1994.

HUBERMAN, A M.Comose realizam as mudanças em educação - subsídios para o estudo da inovação.São Paulo: Altrex, 1972.

MARTINS, Geraldo e GALVÃO, Gerson. O Diretório dos grupos de pesquisa no Brasil: perspectivas de fomento e avaliação.Educação Brasileira, Brasília, v. 16, n. 33, p.11-29, 1994.

MIRANDA, Marília Gouvea de. Novo paradigma de conhecimento e políticas educacionais na América Latina. Cadernos de Pesquisa, São Paulo, v. 100, p. 37-48, 1997.

STUMPF, Ida Regina Chitto. Revistas universitárias brasileiras: barreiras na sua produção. Transinformação Campinas, v. 9, n. 1, p. 34-48, 1997.

SODEK, Elbe Benetti et al. Títulos de dissertações de mestrado: PUC-Campinas e UFMG (1990/1994) 
Transinformação, Campinas, v. 9, n. 1, p. 80-92, 1997.

TARAPANOFF, Kira. A educação superior dentro dos planos do governo. Educação e realidade - a modernidade o sujeito a educação. Porto Alegre, Atica, v. 17, n. 2, p. 92-107, 1992.

TIRAMONTE, Guilhermina. O cenário político e educacional dos anos 90: a nova fragmentação.
Cadernos de Pesquisa - Fundação Carlos Chagas. São Paulo, v. 100, p.79-91, 1997.

WITTER, Geraldina Porto. Pesquisa bibliográfica, pesquisa documental e busca de informação. Estudos de Psicologia, Campinas, v. 5, n. 1, p. 70-83, 1990a.

. Pesquisa como processo de tomada de decisão: variáveis relevantes. Revista de Biblioteconomia de Brasília, v. 18, n. 1, p. 41-58, 1990 b. 